\title{
Study on Elementary Process of Adhesive Wear Using Scanning Probe Microscopy
}

\author{
Alan Hase ${ }^{1)^{*}}$ and Hiroshi Mishina ${ }^{2)}$ \\ ${ }^{1)}$ Department of Mechanical Engineering, Saitama Institute of Technology \\ 1690 Fusaiji, Fukaya, Saitama 369-0293, Japan \\ ${ }^{2)}$ Department of Artificial System Science, Graduate School of Engineering, Chiba University \\ 1-33, Yayoi, Inage, Chiba 263-0022, Japan \\ *Corresponding author: alan_hase@sit.ac.jp
}

( Manuscript received 28 August 2015; accepted 22 December 2015; published 30 April 2016 )

(Presented at the International Tribology Conference Tokyo 2015, 16-20 September, 2015 )

\begin{abstract}
In the elementary process of adhesive wear, wear elements, which are the elemental debris of wear particles, are generated at junctions of asperities, and subsequently grow into transfer particles between sliding surfaces through a mutual transfer and growth process. To elucidate the mechanism of adhesive wear and to establish a wear equation, it is necessary to investigate the characteristics of wear elements and transfer particles in more detail. In this study, changes in the numbers and shapes of wear elements for various metals were examined by means of scanning probe microscopy. In addition, the relationship between the numbers of wear elements and the adhesion forces, determined by friction force microscopy and force-curve measurements, was examined to evaluate the probability of generation of wear elements. The findings on the elementary processes of adhesive wear obtained from this study should be useful in establishing a future theory of wear.
\end{abstract}

Keywords: adhesive wear, wear elements, scanning probe microscopy, friction force microscopy, force curve

\section{Introduction}

Adhesive wear, one of the main mechanisms of wear, involves complex phenomena, as it is affected many factors such as the nature of the materials, the sliding conditions, and the presence of substances between the sliding surfaces. In the elementary process of adhesive wear, wear particles originate from wear elements generated at the actual contact area. Observations made by atomic force microscopy (AFM) have shown that the size of wear elements is of the order of nanometers [1]. In addition, in ferromagnetic materials, the generation of tiny wear elements with a size similar to that of a single magnetic domain has been confirmed by means of magnetic force microscopy (MFM) [2]. From these experimental results, we have proposed the model for the elementary process of adhesive wear that is shown in Fig. 1; this is based on Sasada's wear model [3], with some updates. Although studies on the mechanism of wear have been made at the atomic scale by means of transmission electron microscopy (TEM) [4-6], no series of wear processes has been elucidated yet. To elucidate the mechanism of adhesive wear, it is necessary to investigate the characteristics of wear elements and transfer particles in more detail.
Here, we extend our studies on the elementary process of adhesive wear that we discussed in our previous paper [1]. In particular, we focus on the changes in numbers and shapes of wear elements and transfer particles. Moreover, to evaluate the probability of the generation of wear elements, we measured adhesion forces by means of scanning probe microscopy (SPM). The findings on the elementary process of adhesive wear obtained from this study should be useful in establishing a future theory of wear, and their careful examination could lead to new paths in research and development.

\section{Experimental methods and conditions}

2.1. Micro-sliding friction tests and AFM observations

Friction and wear experiments were executed by using a pin-on-block-type micro-sliding friction tester of our own design, as shown in Fig. 2(a). The nose shape of the pin is a hemisphere of diameter $4 \mathrm{~mm}$. The size of the block specimen is $30 \times 15 \times 10 \mathrm{~mm}$. Seven different metals were used as pin specimens: iron, aluminum, copper, silver, lead, cobalt, and nickel. These seven pin materials in the experiments were slid once on a block specimen of iron by using a piezoelectric actuator. The properties of the materials used in the micro-sliding 


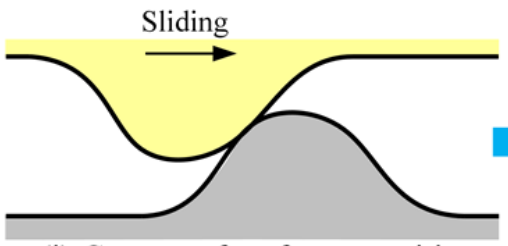

(i) Contact of surface asperities.

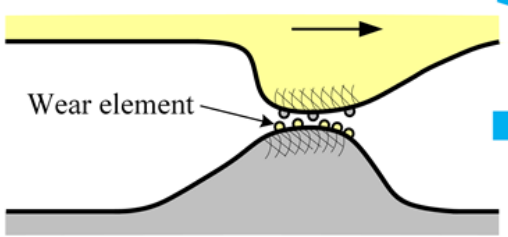

(iii) Wear elements are generated by the transfer of fine structures enclosed by slip lines.

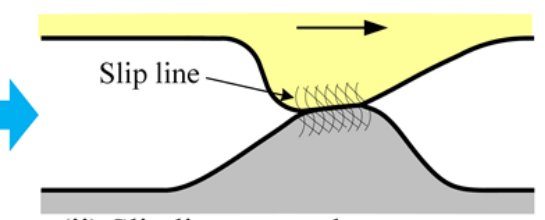

(ii) Slip lines occur by contact stress. A junction is formed.

Fig. 1 Model for the generation of wear elements and transfer particles

friction tests are summarized in Table 1. The experiments were performed under a normal load $W=$ $0.1 \mathrm{~N}$ and at a sliding velocity $v=120 \mu \mathrm{m} / \mathrm{s}$. The sliding distance $L$ was $120 \mu \mathrm{m}$. The surface of both specimens was finished to less than $R_{\max }=50 \mathrm{~nm}$ by mechanical polishing. The pin was slid vertically against the direction of polishing. All the experiments were repeated under dry conditions in air at room temperature (about $20^{\circ} \mathrm{C}$ ) and ambient relative humidity (about $40 \%)$.

After the friction and wear experiments, the block specimen was thoroughly washed with acetone, and the surface of the wear track on the block specimen was visualized in the AFM mode of SPM (horizontal resolution: $0.1 \mathrm{~nm}$; vertical resolution: $0.01 \mathrm{~nm}$ ). The tip used in the AFM measurements was made of silicon and had radius of $10 \mathrm{~nm}$. The AFM measurements were made in the tapping mode. The size and quantity of wear elements and transfer particles on the friction surface of the block specimen were evaluated by means of the particle-size distributions determined from the AFM measurements. Also, the shape of the wear elements on the friction surface of the block specimen was evaluated by using the profile curve determined from the AFM measurements. Parameters of the AFM

Table 1 Specification of materials for the micro-sliding friction test

\begin{tabular}{l|l|l|l}
\hline Specimen & Material & $\begin{array}{l}\text { Purity } \\
(\%)\end{array}$ & $\begin{array}{l}\text { Hardness } \\
(\mathrm{HV})\end{array}$ \\
\hline \multirow{5}{*}{ Pin } & $\mathrm{Fe}$ & 99.9 & 96.1 \\
\cline { 2 - 4 } & $\mathrm{Al}$ & 99.99 & 32.4 \\
\cline { 2 - 4 } & $\mathrm{Cu}$ & 99.99 & 79.9 \\
\cline { 2 - 4 } & $\mathrm{Ag}$ & 99.99 & 92.4 \\
\cline { 2 - 4 } & $\mathrm{Pb}$ & 99.9 & 5.7 \\
\cline { 2 - 4 } & $\mathrm{Co}$ & 99.99 & 160 \\
\cline { 2 - 4 } & $\mathrm{Ni}$ & 99.7 & 85 \\
\hline Block & $\mathrm{Fe}$ & 99.9 & 98.7 \\
\hline
\end{tabular}
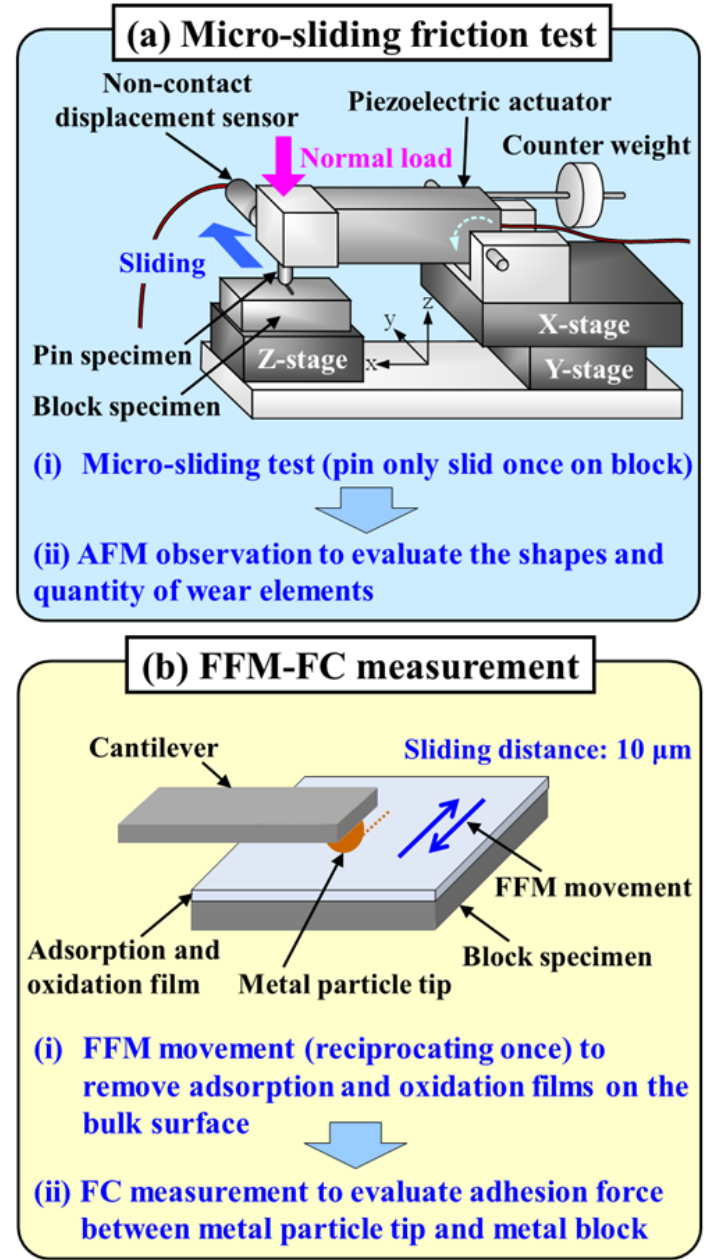

Fig. 2 Schematics showing the experimental setups

measurements are optimized for each sample to obtain a high-resolution image of the block specimen surface before sliding. 
2.2. Friction force microscopy and force-curve measurements

To examine the relationship between the experimental results obtained as described in Section 2.1 and the adhesion force, which one of the major factors controlling adhesive wear, we measured the metal-to-metal adhesion force by means of friction force microscopy (FFM) in combination with force-curve (FC) measurements [4], as shown in Fig. 2(b). In these measurements, a metallic colloidal particle was glued to a tip-less cantilever that we built ourselves and which was operated in a similar manner to a normal cantilever. The FFM-FC measurements were performed on the block specimen of iron by using a probe attached the metallic colloidal particle. A force-curve measurement was performed for each scan of the friction force in which the probe was slid back and forth once.

In this study, we measured the metal-to-metal adhesion force for two sets of materials: iron on iron and copper on iron. The properties of the materials used in the FFM-FC measurements are summarized in Table 2. The diameter of the metal particle was about $20 \mu \mathrm{m}$. The size of the block specimen was $30 \times 15 \times 10 \mathrm{~mm}$. The surface of block specimens was finished to less than $R_{\max } 50 \mathrm{~nm}$ by mechanical polishing. Both surfaces were degreased by washing with acetone before each measurement. The sliding distance for each FFM movement was $10 \mu \mathrm{m}$. The FFM-FC measurements were repeated for 50 cycles and the adhesion force was calculated as an average value. The resolution in the force measurements was around $1 \mathrm{nN}$. All the measurements were repeated in vacuum $\left(1.0 \times 10^{-3} \mathrm{~Pa}\right)$ at room temperature (about $20^{\circ} \mathrm{C}$ ).

Table 2 Specification of materials for FFM-FC measurement

\begin{tabular}{l|l|l|l}
\hline Specimen & Material & $\begin{array}{l}\text { Purity } \\
(\%)\end{array}$ & $\begin{array}{l}\text { Hardness } \\
(\mathrm{HV})\end{array}$ \\
\hline $\begin{array}{l}\text { Colloidal particle } \\
\text { (Probe) }\end{array}$ & $\mathrm{Fe}$ & $>99$ & \multirow{2}{*}{ N/A } \\
\cline { 3 - 4 } & $\mathrm{Cu}$ & 99.8 & \\
\hline Block & $\mathrm{Fe}$ & 99.5 & 97 \\
\hline
\end{tabular}

\section{Results and discussion}

\subsection{Quantity, size and shape of wear elements}

Figure 3 shows AFM images of the surface of the iron block before and after rubbing. The results were obtained by rubbing an iron pin on an iron block. The arrow indicates the direction of sliding. The white and black arrowheads indicate regions where wear elements and transfer particles, respectively, are present. Whereas no particles were observed and only a grinding mark was present before rubbing, the generation of wear elements on the friction surface was apparent after rubbing. Also, transfer particles, which are aggregates of wear elements, could be seen. In the particle analysis, a particle that existed as a single particle on the nanometer scale was regarded as a wear element.

In our previous paper [1], we performed experiments to evaluate the quantities and sizes of wear elements for five materials: iron, aluminum, copper, silver, and lead. Two new materials, cobalt and nickel, were added in the current studies. Figure 4 shows the quantity of wear elements per unit area, generated by rubbing the seven different metals on iron, plotted against their adhesion force to iron. The quantity of wear elements per unit area was evaluated from the number of wear elements present in an area of $2.5 \times 2.5 \mu \mathrm{m}$ examined by AFM in each experiment. The error bars correspond to the scatter in measured values of two experiments. The plots labeled with filled circles $(\bullet)$ use the adhesion forces measured by Buckley [7]. The relationships between the quantity of wear elements and the adhesion force obtained by FFM-FC measurements are labeled with filled squares ( $\mathbf{})$ and will be described later. Fig. 4 shows that there is a marked correlation between our older data for five metals and our new data. We can therefore clearly state that the number of wear elements that is generated is directly proportional to the adhesion force.

Figure 5 shows the particle-size distribution curves of wear elements for the seven pin materials. The newly collected results were similar to our previous data. A peak was found in each particle-size distribution curve, although it was uneven. That is to say, there appears to be an intrinsic size of wear elements for each material.

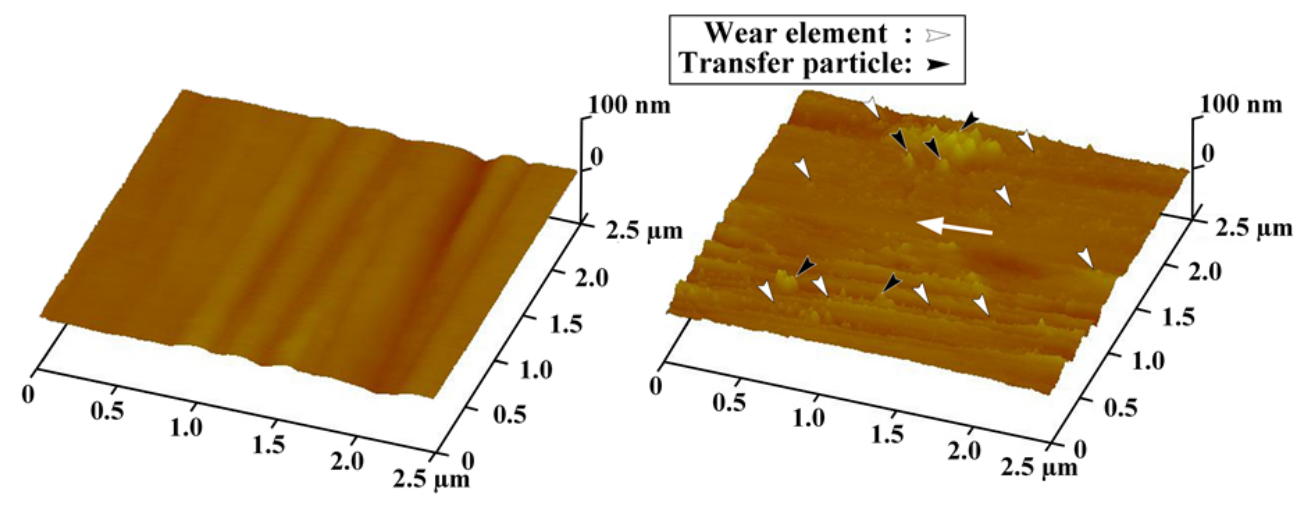

Fig. 3 AFM images of the friction surface of the block specimen before and after rubbing for $\mathrm{Fe} / \mathrm{Fe}$ 


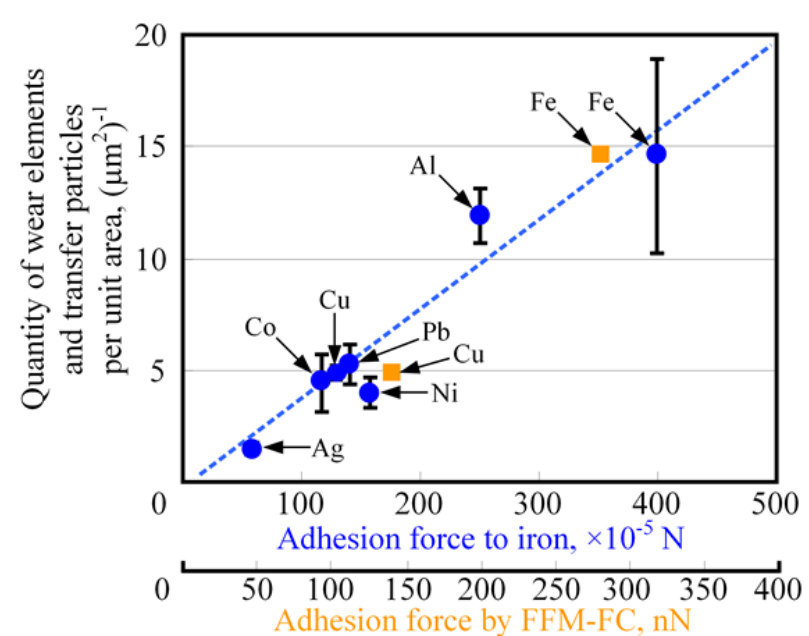

Fig. 4 Quantity of wear elements per unit area, generated by rubbing seven metals on iron, plotted against the adhesion force to iron

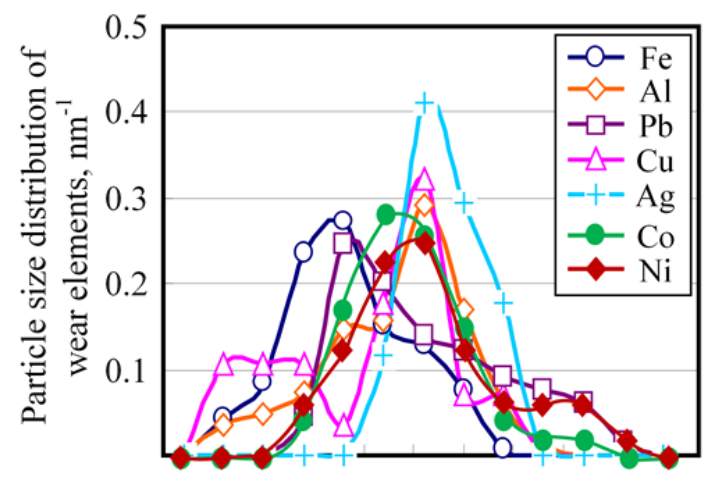

$0 \quad 5 \quad 101520253035404550$

\section{Diameter of the wear element, $\mathrm{nm}$}

Fig. 5 Particle-size distribution curves of the wear elements for seven metals

The intrinsic size for the wear elements of the seven metals used in this study was $10-30 \mathrm{~nm}$ in diameter. Elementary slips on the nanometer scale occur on the surface of a material undergoing plastic deformation [8]. Therefore, one explanation for the size of the wear elements is thought to be the interval of elementary slips [1]. In the AFM studies, in addition to the results discussed above, we did not observe the formation of any holes on the worn surface caused by removal of wear elements. It is likely that any holes that were formed by losses of groups of atoms were subsequently filled by atomic diffusion and plastic deformation [9].

Little is known about the shapes of wear elements. It is interesting to discuss the shapes of wear elements to understand the elementary process of adhesive wear and to establish a wear equation. If wear elements are hemispherical, their diameters should be around 10-30 $\mathrm{nm}$, as can be seen from Fig. 5. To examine their shapes more closely, profile curves of the wear element for $\mathrm{Fe} / \mathrm{Fe}$ and $\mathrm{Ag} / \mathrm{Fe}$ were examined and the results are shown in Fig. 6. From the analyzed data, we found that
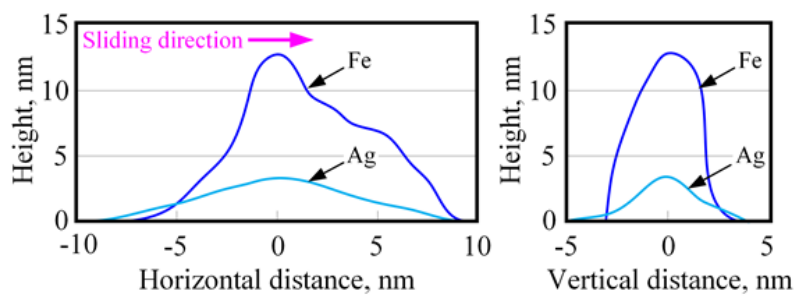

Fig. 6 Profile curves of wear element on the friction surface of the block specimen for $\mathrm{Fe} / \mathrm{Fe}$ and $\mathrm{Ag} / \mathrm{Fe}$

the shapes of wear elements tended to resemble semi-ellipsoids with their major axes aligned to the direction of sliding. This type of shape is formed because as each wear element is removed, it becomes deformed and is stretched along the direction of sliding as a result of friction. The values from profile curves are usually slightly larger than their actual sizes in the outer of particles because the profile curves of AFM data are depends on the curvature radius of the SPM tip used. On the other hand, the curvature radius of the SPM tip does not affect the height of AFM data. In our future work, we will examine the shapes of wear elements using a nanotube tip or a much finer tip to reduce the influence of the shape of AFM tip.

3.2. Measurement of adhesion forces to predict the quantity of wear elements

As discussed in Section 3.1, there is a direct proportional relationship between the quantity of wear elements and the adhesion force between the two materials. Consequently, the probability of generation of wear elements can be evaluated by using this relationship. However, it is difficult to measure metal-to-metal adhesion forces precisely unless a special experimental setup is prepared. Although studies have been made on the measurements of adhesion force by force-curve measurements using a probe with a silicon or silicon nitride tip $[10,11]$, the results correspond to the adhesion forces between the metal specimen and the tip material, and not the adhesion force between two metal specimens. The use of FFM-FC measurements to measure the actual metalto-metal adhesion force has been suggested in a recent study [12]. As the first step in the evaluation of the probability of generation of wear elements, we examined the quantity of wear elements and the adhesion force obtained by FFM-FC measurements for two materials: iron and copper.

The adhesion force measured by FC measurements alone was smaller than that obtained by FFM-FC measurements, because of the effect of adsorbed and oxidized films on the surface of the material. In FFM-FC measurements, the value of adhesion force increased as the number of repeat measurements was increased, and eventually it stabilized after the 15th cycle, when the adsorption and oxidation films had been removed by repeated frictional movements. The average 
adhesion force was therefore determined from the 15 th to the 50th cycles, where the data points were stable. Our two measured value for $\mathrm{Fe} / \mathrm{Fe}$ and $\mathrm{Cu} / \mathrm{Fe}$ (probe/block), shown as filled squares (घ) in Fig. 4, nearly overlapped the correlation line obtained by using Buckley's data. This result clearly shows that wear elements are generated by transfer of materials between the sliding surfaces through adhesion forces; this relationship is apparent even when different methods for measuring the adhesion force are used. We can therefore state that measurement of the adhesion force is a valid method for evaluating the probability of generation of wear elements. Although we obtained only two sets of results by FFM-FC measurements, we intend to perform more studies involving FFM-FC measurements for other materials in the future.

\subsection{Confirmation of the mutual transfer and growth} process by AFM observations

Figure 7 shows AFM images of the surface of the iron block at sliding distances of (a) $30 \mu \mathrm{m}$, (b) $60 \mu \mathrm{m}$ and (c) $90 \mu \mathrm{m}$. Here, the end points of the sliding were not taken into consideration, because an edge deformed by ploughing on the forward movement of the pin specimen was present. Figure 8 shows the rate of change in the quantity of wear elements or transfer particles in different sliding distances for $\mathrm{Fe} / \mathrm{Fe}$, which is corresponding to the data of Fig. 7. Wear elements and transfer particles can be distinguished from their profile curves (side view) and the shape profile (top view) at the particle analysis. We can recognize from Fig. 7 that the quantity of wear elements on the friction surface does not change markedly with the sliding distance. Also, we can see from Fig. 8 that the quantity of transfer particles increases as the sliding distance increases while the quantity of wear elements remain the same or not affected by the increased in the sliding distance. This is because that the probability of generation of wear elements is controlled by the adhesion force between the two materials, as discussed in Section 3.2.

On the other hand, we can see from Figs. 7 and 8 that the quantity of transfer particles tends to increase with increasing sliding distance. The presence of a mutual transfer and growth process was confirmed by macroscopic observations with an optical microscope [13] and a scanning electron microscope [14]. AFM
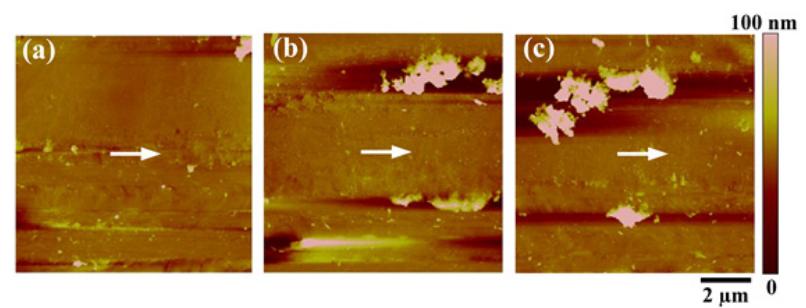

Fig. 7 AFM images of the friction surface of the iron block: (a) sliding distance $L=30 \mu \mathrm{m}$; (b) $60 \mu \mathrm{m}$ and (c) $90 \mu \mathrm{m}$ for $\mathrm{Fe} / \mathrm{Fe}$

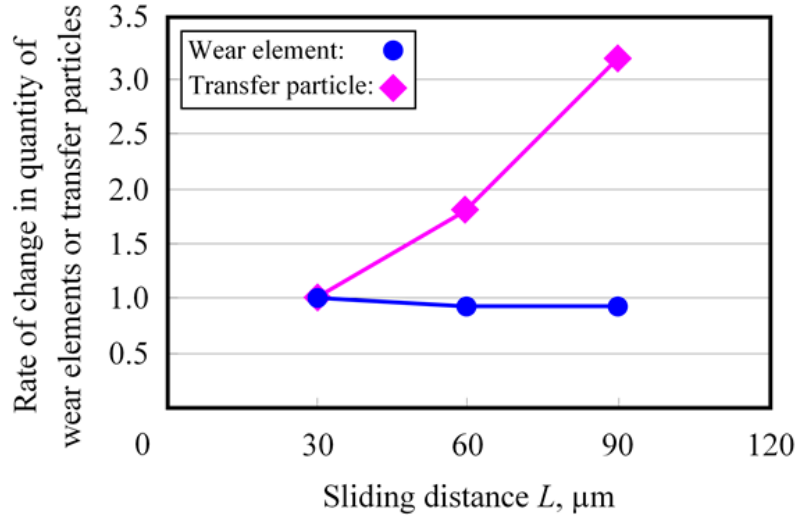

Fig. 8 Rate of change in quantity of wear elements or transfer particles in different sliding distances for $\mathrm{Fe} / \mathrm{Fe}$

observations added further confirmation that transfer particles are formed through a mutual transfer and growth process between sliding surfaces, as shown in Fig. 1.

\subsection{Quantity of transfer particles}

As we have seen above, a transfer particle is formed by the accumulation of wear elements generated between the sliding surfaces. Once a wear element is removed from the friction surface, the adhesion forces acting on it are weakened because atmospheric gas molecules are immediately adsorbed on the surface of the wear element, interrupting direct contacts with the wear surface. In the process of formation of transfer particles, wear elements generated from the two materials aggregate between the sliding surfaces, as shown in Fig. 1(iv). Figure 9 shows profile curve examples for small and large transfer particles on the friction surface of the block specimen for $\mathrm{Fe} / \mathrm{Fe}$. By looking at the height profile curves (also, shape profiles), we can tell which ones are wear elements or transfer particles. Profile curves of transfer particles have multiple peaks, showing aggregation of wear elements.

In our previous paper, we stated that it is possible that the quantity of the transfer particles is controlled by the mutual solubility of the materials. To examine the quantitative relationship between the number of transfer particles and the mutual solubility of the two materials, we plotted the number of transfer particles against the mutual solubility of iron and the various other metals used in the experiments [15]. Figure 10 shows the quantity of transfer particles per unit area formed by rubbing seven metals on iron plotted against their mutual solubility with iron. The quantity of the transfer particles formed is therefore directly proportional to the mutual solubility of the two materials. This is because each transfer particle is formed as a mixture of the two rubbing materials as a result of the mutual transfer and growth process [16]. Finally, whether or not transfer particles are removed as wear particles is controlled by the absorptivity of atmospheric gas molecules on the 
(a)

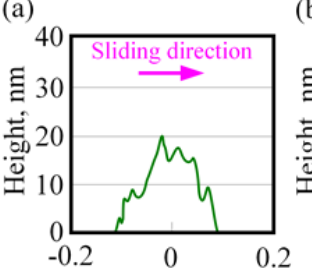

(b)

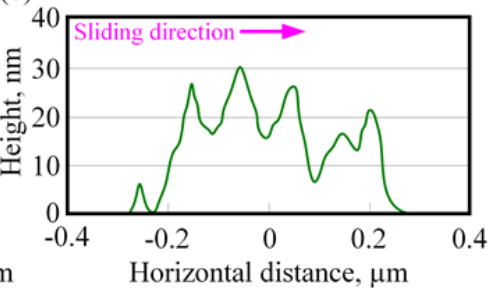

Fig. 9 Profile curves of (a) small transfer particle and (b) large transfer particle on the friction surface of the block specimen for $\mathrm{Fe} / \mathrm{Fe}$

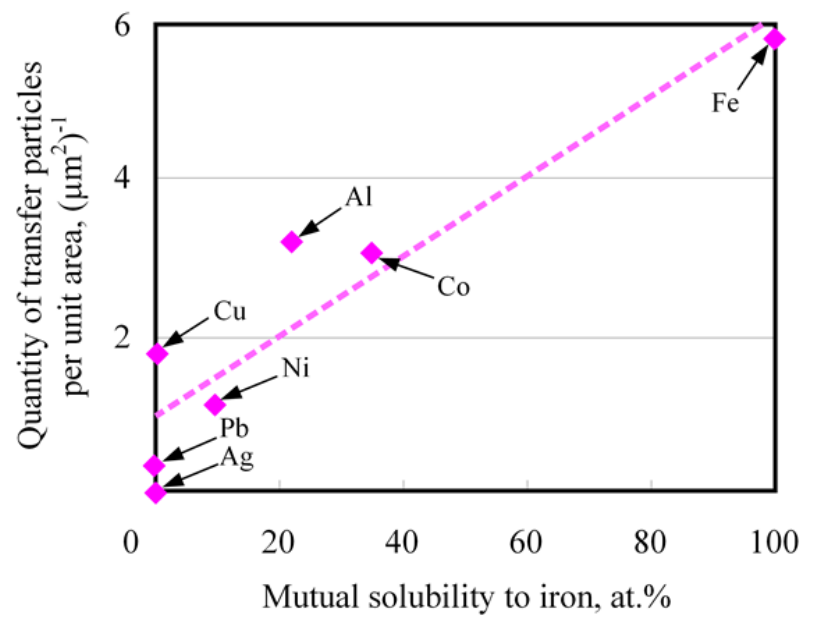

Fig. 10 Quantity of transfer particles per unit area, formed by rubbing seven metals on iron, plotted against the mutual solubility to iron

materials [17-19] and by the magnitude of the moment acting on the transfer particles [20].

Overall, the main factors affecting the elementary process of adhesive wear are the adhesion force and the mutual solubility between the rubbing materials, which are related to the quantity of wear elements and the quantity of transfer particles, respectively. We hope that the path to studies on wear will open as the effects of these factors on the elementary process of adhesive wear become clear.

\section{Conclusions}

We examined the factors that affect the generation, shapes and quantity of wear elements, and their relationships to the adhesion force present during the rubbing process. The factors affecting the formation of transfer particles were also examined. The following conclusions were drawn.

1. The numbers of generated wear elements is directly proportional to the adhesion force between the two materials involved.

2. There is an intrinsic size of wear elements for each material. The intrinsic size of the wear elements for the seven metals examined in this study was $10-30$ $\mathrm{nm}$ in diameter.

3. The adhesion force obtained by FFM-FC measurements can be used to calculate the probability of generation of wear elements.

4. The quantity of transfer particles increases with increasing sliding distance. By means of microscopic AFM observations, we obtained additional confirmation that transfer particles are formed by a mutual transfer and growth process between the sliding surfaces.

5. The quantity of transfer particles that is formed is direct proportional to the mutual solubility of two materials involved.

\section{Acknowledgements}

This research was supported in part by the Japan Society for the Promotion of Science [Grant-in-Aid for Scientific Research (C), No. 26420075]. The authors thank Yamato Ichimoto, who was a student of Chiba University when the FFM-FC measurements were performed, for his assistance.

\section{References}

[1] Hase, A. and Mishina, H., "Wear Elements Generated in the Elementary Process of Wear," Tribol. Int., 42, 2009, 1684-1690.

[2] Mishina, H., Iwase, H. and Hase, A., "Generation of Wear Elements and Origin of Tribomagnetization Phenomenon," Wear, 269, 2010, 491-497.

[3] Sasada, T. and Norose, S., "The Formation and Growth of Wear Particles through Mutual Material Transfer," Proc. JSLE-ASLE Int. Lubrication Conf., Amsterdam 1975, 1976, 82-91.

[4] Jacobs, T. D. B. and Carpick, R. W., "Nanoscale Wear as a Stress-Assisted Chemical Reaction," Nature Nanotechnology, 8, 2013, 108-112.

[5] Sato, T., Ishida, T., Jalabert, L. and Fujita, H., "Real-Time Transmission Electron Microscope Observation of Nanofriction at a Single $\mathrm{Ag}$ Asperity," Nanotechnology, 23, 50, 2012, 505701.

[6] Kizuka, T., "Direct Atomistic Observation of a Contact Process between Crystalline Silicon Surfaces at Room Temperature," Phys. Rev. B, 63, 2001, 033309.

[7] Buckley, D. H., "Adhesion of Single Crystal Metals to Clean Iron (011) Surface Studied by Emission Spectroscopy," NASA Technical Note, D-7018, 1971, 1-22.

[8] Wilsdorf, H., Van der Merwe, J. H. and Kuhlmann-Wilsdorf, D., "Elementary Structure and Slip-Band Formation in Aluminum," Philosophical Magazine, 43, 1952, 632-643.

[9] Kizuka, T., "Atomic Process of Point Contact in Gold Studied by High-resolution Transmission Electron Microscopy,” Phys. Rev. Lett., 81, 1998, 4448-4451. 
[10] Ando, Y., "The Effect of Relative Humidity on Friction and Pull-Off Forces Measured on Submicron-size Asperity Arrays," Wear, 238, 1, 2000, 12-19.

[11] Li, Y. and Li, D. Y., "Experimental Studies on Relationships between the Electron Work Function, Adhesion, and Friction for 3d Transition Metals," J. Appl. Phys., 95, 2004, 7961.

[12] Ichimoto, Y., Kobayashi, H., Arai, S., Omori, T., Mishina, H. and Hase, A., "Metallic Adhesion Force Measurements Using AFM-FC Systems," Proc. JAST Tribology Conference, Fukuoka, October 2013, 2013, A3 (in Japanese).

[13] Sasada, T., Norose, S. and Mishina, H., "The Behavior of Adhered Fragments Interposed Between Sliding Surfaces and the Formation Process of Wear Particles," J. of Lubrication Tech., 103, 2, 1981, 195-202.

[14] Hiratsuka, K., "Environmental Effects on the Formation Process of Adhesive Wear Particles," Tribol. Int., 28, 5, 1995, 279-286.
[15] Booser, E. R., "CRC Handbook of Lubrication," Vol.2 CRC Press, 1984, 204.

[16] Mishina, H. and Sasada, T., "Observation of Micro-Structure in Seized Portion and Mechanism of Seizure," J. Tribol., 108, 1, 1986, 128-133.

[17] Buckley, D. H., "Influence of Chemisorbed Films of Various Gases on Adhesion and Friction of Tungsten," Journal of Applied Physics, 39, 9, 1968, 4224-4233.

[18] Mishina, H., "Chemisorption of Diatomic Gas Molecules and Atmospheric Characteristics in Adhesive Wear and Friction of Metals," Wear 180, 1995, 1-7.

[19] Hiratsuka, K. and Meki, Y., "The Effects of Non-Friction Time and Atmosphere in Friction/Non-Friction Areas on the Wear of Metals," Wear, 270, 7-8, 2011, 446-454.

[20] Sasada, T., "Formation of Spherical and Roller-Like Wear Particles," Report of the Chiba Institute of Technology, 46, 1999, 49-56 (in Japanese). 